\title{
Phoneme monitoring and lexical processing: Evidence for associative context effects
}

\author{
ULI H. FRAUENFELDER \\ Max-Planck-Institut für Psycholinguistik, Nijmegen, The Netherlands \\ and \\ JUAN SEGUI \\ Laboratoire de Psychologie Expérimentale, associé au CNRS, Paris, France
}

\begin{abstract}
In this paper, we propose a new version of the phoneme monitoring task that is well-suited for the study of lexical processing. The generalized phoneme monitoring (GPM) task, in which subjects detect target phonemes appearing anywhere in the test words, was shown to be sensitive to associative context effects. In Experiment 1, using the standard phoneme monitoring procedure in which subjects detect only word-initial targets, no effect of associative context was obtained. In contrast, clear context effects were observed in Experiment 2, which used the GPM task. Subjects responded faster to word-initial and word-medial targets when the target-bearing words were preceded by an associatively related word than when preceded by an unrelated one. The differential effect of context in the two versions of the phoneme monitoring task was interpreted with reference to task demands and their role in directing selective attention. Experiment 3 showed that the size of the context effect was unaffected by the proportion of related words in the experiment, suggesting that the observed effects were not due to subject strategies.
\end{abstract}

Psycholinguists interested in understanding the mental processes and representations underlying lexical processing face a major dilemma. How is it possible to study these hidden and transient mental phenomena while minimally affecting their natural course? In this paper, we investigate the utility of the phoneme monitoring technique for such studies in light of these constraints.

It commonly has been assumed that patterns of detection latencies obtained with the phoneme monitoring technique support inferences about many different aspects of language processing. In its initial application (Foss, 1969), the phoneme monitoring technique was used to gather information about the mental load required to process language at different levels and moments in time. The results of these experiments were used to draw conclusions about processing at the syntactic (Foss \& Lynch, 1969), semantic (Foss \& Jenkins, 1973; Morton \& Long, 1976), and lexical (Foss, 1975) levels. In each case, the assumption was made that processing resources are shared between the phoneme identification process and computations at these higher levels. As more resources are allocated to the higher level computations, the phoneme detection process is slowed down, thereby giving a measure of the complexity of the higher level processes.

\footnotetext{
We would like to thank Guylène Gerard for her help in preparing and running the experiments presented in this paper. This research was supported by Grant ATP 955207 from the CNRS to Juan Segui, who also thanks the Max-Planck-Institut for its generous support during his stays in Nijmegen. Requests for reprints should be sent to Uli Frauenfelder, Max-Planck-Institut für Psycholinguistik, Wundtlaan 1, NL-6525 XD Nijmegen, The Netherlands.
}

More recently, the focus of phoneme monitoring research has shifted to investigations of the lower level processes involved in speech perception and word recognition. In order to study these processes with this task, it has become evident that we need to better understand the nature of the phoneme detection process itself. In particular, research has aimed at determining the type of information used in phoneme identification. Three proposals have been made concerning the representations used in this task.

According to the first, subjects respond to target phonemes only after having recognized the word in which the target is embedded (Foss \& Swinney, 1973; MarslenWilson, 1984; Morton \& Long, 1976). In one such view, the phonological representation that is stored in the mental lexicon is necessarily consulted in making the response. According to the second proposal (Foss \& Gernsbacher, 1983), subjects base their responses only on the acoustic-phonetic representation computed from the signal, and they do not exploit lexical information. Finally, it has been argued that information from both levels plays a role in phoneme identification. According to an autonomous view (Cutler \& Norris, 1979; Foss \& Blank, 1980), subjects can detect phonemes on the basis of two different representations or codes: a prelexical code that is computed directly from the sensory input and a lexical code that is stored in the lexicon. These two codes are elaborated independently, and the response is delivered by the code that first becomes available. If the prelexical code is produced prior to the completion of lexical access, the response is based solely upon this code. Inversely, if lex- 
ical access is achieved before the delivery of the prelexical code, the response is based on the lexical code. An interactive view (McClelland \& Elman, 1986; Stemberger, Elman, \& Haden, 1985), in contrast, holds that subjects respond solely on the basis of the phonemic units of representation, whose activation is influenced by the lexical level. This feedback from the lexical to phonemic level increases as the lexical units containing the target phoneme become more activated. The presence or absence of lexical effects in phoneme monitoring latencies depends upon the amount of lexical feedback; lexical effects emerge only when the lexical level contributes sufficiently to the target phoneme's activation.

It appears now that only this third class of models (either autonomous or interactive), in which both lexical and prelexical information contribute to the phoneme detection process, can account for the large number of otherwise contradictory experimental results found in the phoneme monitoring literature (for a more detailed discussion, see Cutler, Mehler, Norris, \& Segui, 1987). The fact that both prelexical and lexical information contribute to the detection response suggests that phoneme monitoring constitutes a useful tool for studying speech perception and lexcial access. However, the effective use of this procedure in studying lexical processing depends upon being able to establish how and when lexical information intervenes in the phoneme detection response.

Unfortunately, the most widely used version of the phoneme monitoring task, in which subjects are required to detect only targets that constitute the initial phoneme of target-bearing items, is weakly sensitive to lexical processing. Indeed, the identification of the item-initial phoneme targets in this "standard version" is generally made on the basis of prelexical information before lexical information has become available. An examination of the experimental literature reveals that the lexical contribution to phoneme detection in this task is variable and appears to be restricted to specific experimental conditions (see Cutler \& Norris, 1979, and Segui \& Frauenfelder, 1986, for discussions of these conditions). Consider, for example, the effect of lexical status on the detection of targets in monosyllabic items (words vs. nonwords). Cutler et al. (1987) found that the effects of lexical status (detection times to targets in words faster than those to targets in nonwords) appeared and disappeared as a function of the composition of the experimental list (i.e., list monotonymixed lists composed of monosyllabic and bisyllabic items vs. lists of only monosyllabic items). Given the limited and variable sensitivity of this standard procedure to lexical variables, it is important to find another version of the phoneme monitoring task that taps more reliably into lexical processing.

In this paper, we show that the standard phoneme monitoring procedure can be modified to provide a more faithful indicator of the mental processes underlying spoken word recognition. This modification involves having the subjects respond to targets that appear anywhere in the target-bearing word rather than only to targets lo- cated at the beginning of target-bearing items, as is the case in the standard procedure. In the experiments presented below, we contrast this generalized phoneme monitoring (GPM) procedure with the standard one in examining the role of associative semantic context in the determination of phoneme monitoring latencies. We decided to explore such context effects since they constitute one of the most robust effects in lexical processing. Indeed, since the pioneering research of Meyer and Schvaneveldt (1971), a vast number of experiments has confirmed the effect of associative lexical contexts on visual word recognition. This effect (in which the presentation of a word such as doctor facilitates the subsequent recognition of an associatively related word such as nurse) has been obtained using tasks as diverse as lexical decision, naming, and identification thresholds. Our objective in this study was to investigate these context effects in spoken rather than written word recognition by means of the phoneme monitoring task. Furthermore, we aimed to establish the conditions under which this task is sensitive to associative context effects.

\section{EXPERIMENT 1}

In this experiment, we used the standard phoneme monitoring task to determine whether the detection of a target phoneme located in the initial position of the targetbearing word was affected by the prior presentation of a semantically associated word. We also introduced length of the target-bearing word as a variable, since this is one of the factors that can condition the type of information used by subjects in making their phoneme detection responses. In particular, previous research has shown that, at least under certain experimental conditions, an effect of lexical status (targets detected faster in words than in nonwords) is obtained for monosyllabic items (Cutler et al., 1987; Rubin, Turvey, \& van Gelder, 1976), but not for bisyllabic items (Segui, Frauenfelder, \& Mehler, 1981). If the associative context affects the detection response, then we would expect these effects to be larger for monosyllabic than for bisyllabic words.

\section{Method}

Materials. Two experimental lists, each consisting of 160 openclass words (nouns, adjectives, and verbs), were created. Each list contained 20 target-bearing test words ( 10 monosyllabic and 10 bisyllabic words). Half of these words were immediately preceded by a semantically associated word, and the other half by nonrelated words. The target-bearing word were the same in the two lists. The two lists were counterbalanced with respect to the context words such that a given target-bearing word was preceded by a related word in one list and by a nonrelated word in the other list. Each word appeared only once in each list.

The semantically associated words were selected on the basis of French word association norms (Lieury, Iff, \& Duris, 1976). They always corresponded to the first associate given by the majority of the subjects. A target phoneme $(/ \mathrm{p} /, / \mathrm{t} /, / \mathrm{k} /$, or $/ \mathrm{d} /)$ was the initial phoneme of each of the 20 test words. The 40 related and unrelated context words were matched in syllabic length. These context words also were selected such that their initial phonemes were 
roughly the same phonological distance (in terms of distinctive features) from the target phoneme, to control for possible phonological interference effects (Stemberger et al., 1985).

In addition to the 20 target-bearing test words and the 20 semantically associated or unrelated context words, each list contained 120 filler words. Sixteen of these fillers also contained a target phoneme in medial position and were bisyllabic. Eight of these words were preceded by a related word and the other 8 by an unrelated one. The remaining filler words were all unrelated. All the words included in the experiment (target-bearing words, context words, and fillers) were either monosyllabic or bisyllabic.

Procedure. The experimental lists were read and recorded (on an Ampex AG-440B tape) by a French native speaker in a continuous fashion at a rate of one word per second. A pulse, inaudible to the subjects, was placed electronically on the burst of the stop consonant targets and was verified visually on the screen of an oscilloscope. These pulses triggered a clock, which was stopped by the subject's response.

Each experimental list was divided into four separate blocks, one for each of the target phonemes to be detected. The order of presentation of the blocks was counterbalanced across subjects. At the beginning of each block, the subjects were given the phoneme to be detected auditorily (for instance, /p/ as in Paris). In addition, the letter corresponding to the target phoneme was presented by means of a small index card. The subjects listened over stereo headphones to the experimental blocks, which consisted of a continuous and equally spaced sequence of words. They were instructed to respond as rapidly as possible by pushing a response key with their dominant hand when they heard the previously specified target phoneme in word-initial position.

Subjects. Two groups of 22 subjects, students at the University of Paris V, were tested. Each group received one of the two experimental lists (L1 or L2).

\section{Results}

All reaction times (RTs) above $1,000 \mathrm{msec}$ and below $100 \mathrm{msec}$ were eliminated from the analysis. The eliminated responses represented only $2.2 \%$ of the total data. The mean RT for the semantically associated context was $420 \mathrm{msec}$ (414 and $426 \mathrm{msec}$ for the monosyllabic and bisyllabic conditions, respectively), and that for the semantically unrelated context was $421 \mathrm{msec}$ (418 and $425 \mathrm{msec}$ for the monosyllabic and bisyllabic conditions, respectively).

An analysis of variance showed that neither of the main factors, semantic relation and word length, produced significant differences ( $F<1$ in both cases). The interaction between these factors was also not significant $(F<1)$.

\section{Discussion}

In Experiment 1, we failed to find an effect of associative context upon subjects' phoneme detection latencies. Given the robustness of such context effects found with other experimental procedures, we cannot exclude the possibility that the context did affect the identification of the target-bearing words, but not the phoneme monitoring response. The context word may well have accelerated lexical access to the target-bearing word, but not enough to make the lexical code available before a response based on the prelexical code was delivered. The absence of an associative context effect confirms that the standard procedure does not provide a very reliable indicator of lexical processing.

Given this state of affairs, it is important to find an alternative version of the phoneme monitoring task that reflects lexical processing more faithfully. The GPM procedure, in which targets appear anywhere in targetbearing words, constitutes a good candidate. In Experiment 2, we used this modified version of phoneme monitoring with the same experimental tapes used in Experiment 1 in order to compare it with the standard procedure. These tapes included word-internal targets, to which the subjects in Experiment 1 of course did not respond, but which in this experiment should permit us to gather information concerning the evolution of these lexical context effects across time. We expected these effects to increase as the target arrived later in the target-bearing word, since lexical processing of this word would have progressed farther.

\section{EXPERIMENT 2}

\section{Method}

Materials. The same experimental materials and lists were used as in Experiment 1. However, subjects responded not only to the 20 word-initial targets, but also to the 8 test items with word-medial targets. These test items were counterbalanced across lists so that half were preceded by associatively related words and the other half by nonrelated words. Table 1 gives examples of monosyllabic stimuli (with the target phoneme /p/ in item-initial position) and bisyllabic stimuli (with the target phoneme $/ t /$ in medial position).

Procedure. The experimental procedure used in Experiment 1 was employed; however, the subjects were given a different instruction set. They were instructed to react to target phonemes located anywhere in the target-bearing word and not only to those in wordinitial position as in the previous experiment.

Subjects. Two groups of 20 subjects, students at the University of Paris V, were tested. Each group received one of the two experimental lists ( $\mathrm{L} 1$ or $\mathrm{L} 2$ )

\section{Results}

For the analysis of the data, we eliminated all reaction times above $1,000 \mathrm{msec}$ and below $100 \mathrm{msec}$. The eliminated RTs represented only $2.4 \%$ of the total responses. The mean RTs for semantically related words with initial and medial targets were 491 and $438 \mathrm{msec}$, respec-

Table 1

Examples of Monosyllabic and Bisyllabic Stimuli Used in Experiment 2

\begin{tabular}{|c|c|c|c|c|}
\hline \multirow[b]{3}{*}{ Target Position } & \multicolumn{4}{|c|}{ Associative Relation } \\
\hline & \multicolumn{2}{|c|}{ Related } & \multicolumn{2}{|c|}{ Unrelated } \\
\hline & Prime & Target & Prime & Target \\
\hline Word-Initial & $\begin{array}{l}\text { clou } \\
\text { (nail) }\end{array}$ & $\begin{array}{l}\text { pointe } \\
\text { (point) }\end{array}$ & $\begin{array}{l}\text { cuve } \\
\text { (tank) }\end{array}$ & $\begin{array}{l}\text { pointe } \\
\text { (point) }\end{array}$ \\
\hline Word-Medial & $\begin{array}{l}\text { oreille } \\
\text { (ear) }\end{array}$ & $\begin{array}{l}\text { entendre } \\
\text { (hear) }\end{array}$ & $\begin{array}{l}\text { ressort } \\
\text { (spring) }\end{array}$ & $\begin{array}{l}\text { entendre } \\
\text { (hear) }\end{array}$ \\
\hline
\end{tabular}


tively. For semantically unrelated words, mean RTs were 510 and $473 \mathrm{msec}$ for initial and medial targets, respectively.

An analysis of variance with subjects as the random factor showed that the list factor neither introduced significant differences nor interacted with the main factors. This analysis did reveal a significant effect of semantic relation $[F(1,38)=14.25, p<.001]$ and of target position $[F(1,38)=18.9, p<.001]$. The interaction between these two factors was not significant $[F(1,38)=1.5]$. A Student's $t$ test taking the items as random factor revealed a significant effect of the semantic relation $[t(28)=1.74$, $p<.05$, one-tailed]. When examined separately for each of the two positions, the effects of semantic relation were significant for the initial targets $[F(1,38)=5.39$, $p<.05]$ and for the medial targets $[F(1,38)=9.77$, $p<.005]$. The mean RTs for semantically related monosyllabic and bisyllabic words were 475 and $501 \mathrm{msec}$, respectively. For semantically unrelated monosyllabic and bisyllabic words, the mean RTs were 498 and $513 \mathrm{msec}$, respectively.

An analysis of variance showed that word length introduced a significant effect $[F(1,38)=5.60, p<.02]$, but that this factor did not interact significantly with the associative relation. However, a matched $t$ test revealed an associative context effect for monosyllabic items $[t(39)$ $=1.80, p<.05$, one-tailed], but no such effect for bisyllabic items $[t(39)=0.60]$.

\section{Discussion}

The results of this experiment using the GPM procedure present a striking contrast with those of Experiment 1 . Using the same stimulus tapes, but changing the instructions given to subjects, we obtained an effect of lexical context for targets in word-initial position. RTs were faster when the target-bearing item was preceded by a semantically associated word than when it was preceded by an unrelated one. Furthermore, a facilitatory context effect also was found for targets in word-medial position. These effects of associative lexical context, as well as the more detailed pattern of results to be discussed below, show the sensitivity of the GPM procedure to lexical processing.

The additional finding of an overall RT advantage of word-medial over word-initial targets is suggestive of a lexical contribution to the phoneme detection process elicited by the GPM procedure. The later within a word the target appears, the more the processing of this word has progressed, and consequently the greater the impact of the lexical code. This RT difference across target position must be interpreted with caution, since the targetbearing words for the two positions were different. However, there exists independent evidence for increasing lexical contribution with later target positions. Detection latencies to targets placed in four different positions in matched words and nonwords were compared in an experiment using the GPM procedure (Frauenfelder, Segui, \& Dijkstra, 1987). The difference in the detection times to targets appearing in words and nonwords in the same sequential position and the same local phonemic environment increased as the targets appeared later in their respective target-bearing items. These results show that it is not simply a serial position effect, but rather an effect of lexical processing, that is being measured. This conclusion is further strengthened by other monitoring research (Marslen-Wilson, 1984) in which targets were located at several different positions in isolated words. Detection times for target phonemes varied as a function of their physical distance from the recognition point of the word. The recognition point was determined empirically in a gating study and corresponded to the amount of acoustic information required for $80 \%$ of the subjects to correctly identify the word. The strong correlation between RTs and this distance suggests that phoneme detection depends upon the time-course of lexical access, or, more precisely, the moment at which the lexical code of the target-bearing word becomes available.

Although the lexical contribution to phoneme detection latencies increased as the target arrived later in the targetbearing item, it is important to recall that the effects of the associatively related context word were not restricted to word-internal targets. Even responses to word-initial targets were affected by the associative context, albeit somewhat less than were responses to word-internal targets. In fact, the observed trend toward larger context effects for word-internal than for word-initial targets is also consistent with increased lexical contribution as a function of target position. For word-internal targets, lexical processing is more advanced, making the lexical code more available. As a consequence, the facilitatory influence of the associatively related context word is more likely to manifest itself as an RT advantage.

Closer examination of the results for word-initial targets also revealed a pattern that is expected if the detection process depends upon lexical processing. A context effect was obtained for targets at the beginnings of monosyllabic, but not bisyllabic, words. The lexical code is more likely to be available when the detection response is initiated for monosyllabic words, since they are usually recognized more quickly than are bisyllabic ones.

We can now ask why context effects were found for the word-initial targets in the GPM procedure and not in the standard version. To interpret this differential effect of associative context, we propose that the subjects' reliance upon the prelexical and the lexical codes varies depending upon the instruction set. When given the instruction to monitor for only word-initial phonemes, subjects can focus their attention upon the beginning part of the stimulus and respond rapidly upon the basis of the prelexical code before the lexical code becomes available. The situation is very different for subjects instructed to detect targets appearing anywhere in words. This instruction gives subjects much less information, not only about the location of the target, but also about its nature since the acoustic manifestation of any target phoneme varies considerably as a function of its left and right phonetic 
context. Since subjects are uncertain about the nature and the position of the target, they tend to rely more heavily upon the stored lexical code than upon the prelexical code. Our interpretation of the absence of context effects under the "initial" instruction set attributes a central role in phoneme detection to attention. We return to a consideration of the role of attention in the General Discussion.

We have argued here that the difference in sensitivity to lexical processing between the two procedures used in Experiments 1 and 2 lies in the instruction set and its role in directing attention. There are, however, other differences in the two experiments that could have played a role. In particular, although the experimental lists in the two experiments were identical, subjects actually responded to more items in the GPM experiment (since they responded to the word-internal targets as well as to the word-initial ones). It is feasible that the number of responses to targets preceded by associatively related words could influence the subjects' behavior. Generally, as the number of related items increases, subjects are more likely to notice the relationship between such items and to exploit this relationship in making their responses. Such an explanation, which appeals to subject strategies, appears improbable given the small differences between the two experiments. There are two further reasons why it is unlikely that subjects noticed the associative relationship between words in either experiment. First, only a small proportion of the items in either experiment were related. Second, the words were presented in continuous lists rather than pairwise, as is done in most research investigating context effects in lists. Compared with pairwise presentation of context and target words, continuous presentation of words makes it harder for the subject to identify which words go together and to discover the relationship between them.

Nonetheless, to ensure that the context effects found here with the GPM procedure were not strategic, we conducted a further experiment in which we manipulated the number of associatively related words. If the observed context effects are due to strategies, then we would expect larger effects of context as the number of related words in the experimental list increases (see den Heyer, Briand, \& Dannenbring, 1983; Seidenberg, Waters, Sanders, \& Langer, 1984; Tweedy, Lapinski, \& Schvaneveldt, 1977).

\section{EXPERIMENT 3}

\footnotetext{
Method

Materials. We defined two experimental conditions that varied in the proportion of associatively related words. Four experimental lists, two for the high-proportion condition (HPC) and two for the low-proportion condition (LPC), were constructed. Each list consisted of 120 open-class words and contained 18 bisyllabic test words with the target phoneme in medial position and 6 bisyllabic filler words with the target phoneme in initial or final position. The target was always the phoneme $/ t /$. As in the previous experiments, these test words were counterbalanced across lists with respect to their context word, such that they were preceded by a related word in one list and by an unrelated word in the other. The 6 target-bearing
}

filler words were all preceded by a related word in the HPC and by an unrelated word in the LPC. There was approximately a factortwo difference between the two conditions in the proportion of targetbearing items that were preceded by an associatively related word (63\% for the HPC and $37 \%$ for the LPC).

The number of related filler items without target phonemes was also manipulated. Twenty-six filler words were preceded by an associatively related word in the lists for the HPC condition, as opposed to only 12 filler words in the LPC lists. Overall, the HPC experimental list contained 41 words (with or without targets) preceded by an associately related word, whereas the LPC contained only 20 such items. The semantically related words were selected on the basis of French word association norms (Lieury et al., 1976).

Experimental Procedure. The experimental procedure was identical to that used in Experiment 2.

Subjects. Two groups of $\mathbf{4 0}$ students at the University of Paris V were randomly assigned to two proportionality conditions, HPC and LPC. Subjects within each condition received either List 1 (L1) or List 2 (L2).

\section{Results}

The RT analysis was restricted to the 18 target-bearing test words that were the same in the two proportionality conditions. All reaction times above $1,000 \mathrm{msec}$ and below $100 \mathrm{msec}$ were eliminated from the analysis. The eliminated RTs represented $1.2 \%$ of the total responses. The mean RTs for semantically related words in HPC and LPC were 411 and $421 \mathrm{msec}$, respectively. For semantically unrelated words in HPC and LPC, mean RTs were 450 and $456 \mathrm{msec}$, respectively.

An analysis of variance revealed a significant effect for the associative semantic relation, with (1) subjects and (2) items taken as the random factor $\left[F_{1}(1,76)=20.5\right.$, $p<.001$, and $\left.F_{2}(1,17)=12.7, p<.01\right]$. In contrast, there was no effect of the proportion condition $(F<1$ in both analyses). The interaction between these two factors was also not significant. When analyzed separately for each of the two experimental conditions, the effects of associative semantic relation were significant for both subjects and items $(p<.02$ in both cases.)

\section{Discussion}

This experiment clearly confirms the existence of associative context effects for word-medial targets using a larger number of experimental items than in Experiment 2 . The overall facilitatory effect $(37 \mathrm{msec})$ was comparable in size to that obtained in Experiment 2 (35 msec) for the same target position. In addition, there was no interaction between the context effect and the proportionality conditions; the size of the context effect was almost identical in the two proportionality conditions: $39 \mathrm{msec}$ for HPC and $35 \mathrm{msec}$ for LPC. The constant size of the context effects for these two proportionality conditions suggests, as we argue below, that the effects observed are not determined by the subjects' strategies.

\section{GENERAL DISCUSSION}

In this study, we investigated the role of associative semantic context in spoken word recognition by means of two different versions of the phoneme monitoring task. 
Using the standard phoneme monitoring procedure, in which subjects respond only to item-initial targets, we found no context effects. In Experiment 2, using the same stimuli but a modified procedure (the GPM), in which subjects respond to targets appearing anywhere in the word, we observed a clear context effect. Experiment 3 replicated these latter results and showed that the magnitude of this effect was unaffected by the number of associatively related items present in the experimental list.

We have shown in these experiments that the two versions of the phoneme monitoring task are differentially sensitive to lexical processing or, more specifically, to lexical context effects. ${ }^{1}$ Two factors defining the GPM task favor a greater lexical contribution in the detection responses. These are the instruction set that defines for the subject where the targets to be detected are located (e.g., anywhere vs. initial position) and the actual location of the target phoneme in the target-bearing item. Let us turn first to the effect of the instruction set. Subjects performing in the standard (initial position) version can focus their attention upon the initial sounds of the targetbearing word and use this bottom-up information in making their response before lexical access has taken place. In contrast, subjects in the GPM task have no prior information about the position of the target phoneme and cannot attend to a specific part of the stimulus. As a consequence, they tend to rely more heavily upon the lexical code in order to detect target phonemes.

The role of attention in speech perception and word recognition has been investigated in the related area of phoneme restoration. The phoneme restoration effect (Warren, 1970) refers to a perceptual illusion in which listeners report hearing words intact even when a part of these words is replaced by noise. The strength of this perceptual illusion has been shown to depend upon how much prior information subjects have about the position of the restored phoneme and about the lexical identity of the word (Nusbaum, Walley, Carrell, \& Ressler, 1982; Samuel \& Ressler, 1986). The more such information listeners have to help focus attention upon a specific part of a word, the less lexical knowledge influences their response. It has been argued (Samuel \& Ressler, 1986) that although listeners normally direct their attention to the lexical level, under conditions such as those described here they are capable of focusing on the phonemic level, thereby limiting the influence of the lexicon. There is clear convergence between this interpretation and the one we have advanced. In both, it is claimed that when subjects are led by task demands to focus their attention on a specific part of the word, the effect of the lexicon is minimized.

The sensitivity of the GPM procedure to lexical context is due not only to the instruction set, but, as we have already mentioned, also to the sequential position of the target phoneme in the target-bearing word. The later the target phoneme arrives in the word, the further the lexical processing has progressed and the greater the effect of the lexicon.
Having shown the sensitivity of the GPM procedure to associative context effects, we attempted in Experiment 3 to specify more precisely the nature of these effects by manipulating the proportion of related items within the experimental list. Several authors (den Heyer et al., 1983, Tweedy et al., 1977) have taken an observed relationship between the proportion of related trials and the amount of contextual facilitation in lexical decision latencies to reflect subject strategies. As the proportion increases, so does the ability of subjects to consciously take advantage of the greater predictability of words. To the extent that the proportion manipulations in our experiment were adequate ${ }^{2}$ the constant size of the context effect as a function of this manipulation suggests that our effect is not strategic.

Seidenberg et al. (1984) went further in their interpretation of proportionality effects and argued that these effects serve as one indicator of the locus of context effects. These authors compared the effect of varying proportions of related trials in two tasks-lexical decision and naming - and found proportionality effects only for the former. They concluded, on the basis of this result and convergent evidence from other experiments, that lexical decision latencies reflect some processes that take place after lexical access. These postlexical processes are argued to involve the integration of a word with its context or, in the case of lexical decisions, judgments of the subject about the relationship between the context and target words. Postlexical processes are opposed to prelexical processes, which are responsible for decoding the signal to recognize it as a particular word.

It is clearly essential to determine the extent to which the context effects observed with the GPM procedure have a prelexical and/or a postlexical processing locus. Unfortunately, simply showing that the subject has responded using the lexical representation does not allow us to identify the locus of this effect. Indeed, there is an important distinction to be made between the representation (prelexical or lexical) used in making the detection response and the stages of processing (prelexical or postlexical) where the context effects are localized. If the presentation of the prime facilitates access to the lexical representation of the target-bearing word, we can speak of a prelexical locus of these effects. Context effects have a postlexical locus, when, in contrast, it is not the recognition of the target-bearing word that is facilitated, but rather the decision processes taking place after recognition that involve judgments about the relationship between the prime and target-bearing word.

If the interpretation by Seidenberg et al. (1984) of proportionality effects is correct, our results provide some preliminary evidence that the context effects observed in the present study are not attributable to postlexical processes. It is premature on the sole basis of these results to draw general conclusions concerning the locus of these context effects. It is important to verify this interpretation by means of additional experimental findings. Nonetheless, the results obtained in the present paper sug- 
gest that the GPM task provides a promising way of studying lexical processing and evaluating context effects in spoken language understanding. The sensitivity of this task to processes underlying lexical access makes it useful in resolving the psycholinguist's dilemma of how to study the temporal properties of lexical processing.

\section{REFERENCES}

Cutler, A., Mehler, J., Norris, D., \& Segui, J. (1987). Phoneme identification and the lexicon. Cognitive Psychology, 19,14!-177.

CUtler, A., \& NorRIS, D. (1979). Monitoring sentence comprehension. In W. E. Cooper \& E. T. C. Walker (Eds.). Sentence processing: Psycholinguistic studies presented to Merrill Garrett (pp. 113134). Hillsdale, NJ: Erlbaum.

den Heyer, K., Briand, K., \& Dannenining, G. L. (1983). Strategic factors in a lexical decision task. Evidence for automatic attention and attention driven processes. Memory \& Cognition, 11, 374-381

Foss, D. J. (1969). Decision processes during sentence comprehension Effects of lexical item difficulty and position upon decision times. Joumal of Verbal Learning \& Verbal Behavior, 8. 457-462.

Foss, D. J. (1975). On the time-course of sentence comprehension. In F. Bresson \& J. Mehler (Eds.), Current approaches to problems in psycholinguistics. Paris: CNRS.

Foss, D. J., \& BLANK, M. A. (1980). Identifying the speech codes Cognitive Psychology, 12, 1-31.

Foss, D. J., \& Gernsbacher, M. A. (1983). Cracking the dual code Toward a unitary model of phoneme identification. Journal of Ver bal Learning \& Verbal Behavior, 22, 609-632.

Foss, D. J., JENkINs, C. M. (1973). Some effects of context on the comprehension of ambiguous sentences. Journal of Verbal Learning \& Verbal Behavior, 12, 577-588.

Foss, D. J., \& LYNCH, R. H. (1969). Decision processes during sen tence comprehension: Effects of surface structure on decision times. Perception \& Psychophysics, 5, 145-148.

Foss, D. J., \& SWINNEY, D. A. (1973). On the psychological reality of the phoneme: Perception, identification and consciousness. Journal of Verbal Learning \& Verbal Behavior, 12, 246-257.

Frauenfelder, U. H., Segui, J., \& Dijkstra, T. (1987). Lexical effects in phoneme monitoring: Facilitatory or inhibitory? Proceedings of the 11th International Congress of Phonetic Science (pp. 105-107) Tallinn, USSR.

LieurY, A., IFF, M., \& Duris, P. (1976). Normes d'associations verbales. Paris: Laboratoire de Psychologie Expérimentale.

Marslen-Wilson, W. D. (1984). Function and processes in spoken and word recognition. In H. Bouma \& D. G. Bouwhuis (Eds.), Attention and performance: Control of language processes ( $\mathrm{pp} .125$ 150). Hillsdale, NJ: Erlbaum.

McClelland, J. L., \& Elman, J, L. (1986). The TRACE model of speech perception. Cognitive Psychology, 18, 1-86.

Meyer, D. E., \& SChVAneveldt, R, W. (1971). Facilitation in recognizing pairs of words: Evidence of dependence between retrieval operations. Joumal of Experimental Psychology, 90, 227-234.
Morton, J., \& LONG, J. (1976). Effect of word transition probability on phoneme identification. Journal of Verbal Learning \& Verbal Behavior, 15, 43-51.

Nusbaum, H. C.. Walley, A. C. Carrell, T. D., Ressler, W. H. (1982). Controlled perceptual strategies in phoneme restoration (Research on Speech Perception Progress Report 8, 83-103). Bloomington: Indiana University.

Rubin, P., Turvey, M. T., \& van Geloer, P. (1976). Initial phonemes are detected faster in spoken words than in non-words. Perception \& Psychophysics, 19, 394-398.

Samuel, A. G., Ressler, W. H. (1986). Attention within auditory word perception: Insights from the phoneme restoration illusion. Journal of Experimental Psychology: Human Perception \& Performance. 12, 70-79.

Segui, J., \& Frauenfelder, U. (1986). The effect of lexical constraints upon speech perception. In F. Klix \& H. Hagendorf (Eds.), Human Memory \& Cognitive Abilities: Symposium in Memoriam Hermann Ebbinghaus (pp. 795-808). Amsterdam: North-Holland.

Segui, J., Frauenfelder, U., Mehier, J. (1981). Phoneme monitoring, syllable monitoring, and lexical access. British Journal of Psychology, 72, 471-477.

Seidenberg, M. S., Waters, G. S., Sanders, M., Langer, P. (1984). Pre- and postlexical loci of contextual effects on word recognition. Memory \& Cognition, 12, 315-328.

Stemberger, J. P., Elman, J. L., Haden, P. (1985). Interference between phonemes during phoneme monitoring: Evidence for an interactive activation model of speech perception. Joumal of Experimental Psychology: Human Perception \& Pefformance, 11, 475-489.

Tweedy, J. R., Lapinski, R. H., \& Schvaneveldt, R. W. (1977). Semantic context effects on word recognition: Influence of varying the proportion of items presented in an appropriate context. Memory \& Cognition, 5, 84-89.

Warren, R. M. (1970). Perceptual restoration of missing speech sounds. Science, 167, 392-393.

\section{NOTES}

1. We have obtained other evidence for the effect of the instruction set in a recent study (Segui \& Frauenfelder, 1986), in which we found an interaction between the instruction set (target located anywhere vs. initial target) and word frequency. RTs to phonemes located in wordinitial position of monosyllables were affected by the frequency of the target-bearing word only when subjects were given the "anywhere" instruction and not when they received the standard "initial" instruction.

2. Although the measure of proportion of related items used in this experiment for continuous lists is not directly comparable with those for other experiments using pairwise presentation, we can compute the percentage of word-to-word transitions that involve related items. For this experiment, we arrived at approximately the same proportions as the other experiments: $41 / 119$ transitions for the HPC (34\%) and 20/119 for the LPC $(18 \%)$, as opposed to $30 \%$ and $20 \%$, respectively, used in Seidenberg et al. (1984).

(Manuscript received July 23, 1986; revision accepted for publication April 20, 1988.) 